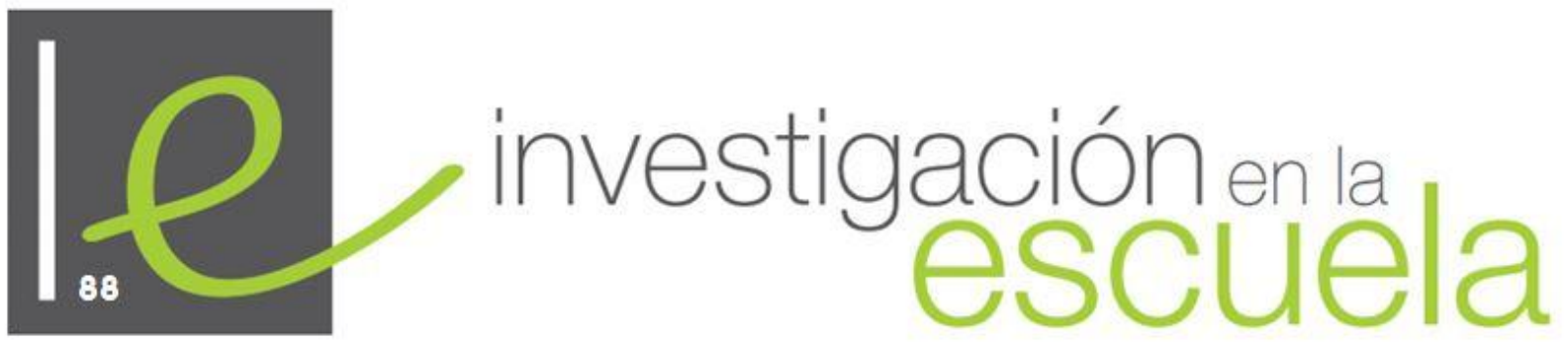

Revista académica evaluada por pares y de acceso abierto

ISSN 2443-9991

\title{
La Enseñanza de la problemática energética para profesorado de ciencias en formación inicial: ¿Conceptos o conciencia?
}

\author{
Carolina Martín Gámez, $M^{a}$ Teresa Prieto Ruz, $M^{a}$ Ángeles Jiménez López. \\ Universidad de Málaga \\ España
}

Citación: Martín, C., Prieto, T. \& Jiménez, M.A. (2016). La enseñanza de la problemática enérgica para profesorado de ciencias en formación inicial: ¿Conceptos o conciencia?. Investigación en la escuela, 88, 78-92. Recuperado de www.investigacionenlaescuela.es/articulos/R88/R88-5

Resumen: en este trabajo se presenta un estudio realizado con profesorado de ciencias en formación inicial, relativo a las características y oportunidades que la problemática energética. Como problema socio-científico, ofrece para promover el aprendizaje de conceptos y el desarrollo de actitudes y valores. Los resultados ponen de manifiesto la predominancia de la idea de que el aprendizaje de los conceptos científicos básicos, es previo e independiente a la toma de conciencia sobre la problemática planteada.

Palabras clave: "profesorado de ciencias en formación inicial"; "problemática energética"; "conceptos"; "actitudes y valores".

Teaching energy issues for pre-service science teachers: Concepts or consciousness? Abstract: this paper presents a study of pre-service science teachers on the features and opportunities that energy issues as socio-scientific problem, offers to promote the learning of concepts and the development of attitudes and values. The results show the predominance of the idea that learning basic scientific concepts is independent prior to the awareness of the issues raised. 
Key words: "pre-service science teachers"; "energy issues"; "concepts”; "attitudes and values".

\section{L'enseignement la problématique de l'énergie pour les professeurs de sciences en formation initiale: Concepts ou conscience?}

Résumé: cet article présente un projet réalisé par des étudiants en dernière année de master destinés à devenir enseignants en sciences, sur les caractéristiques et les possibilités que nous offre la problématique de l'énergie, comme problème socio-scientifique, ceci encourageant l'apprentissage de concepts et le développement d'attitudes et de valeurs. Les résultats mettent en relief l'idée que l'apprentissage de concepts scientifiques de base est préalable et indépendant à la prise de conscience des questions soulevées en rapport à la problématique de l'énergie.

Mosts clé: "formation initial du professorat de sciences"; "les questions énergétiques"; "des concepts"; "des attitudes et des valeurs".

\section{Marco teórico}

En la actualidad, la educación, cualquiera que sea la materia desde la que se aborde, tiene que asumir el reto y la responsabilidad de incorporar perspectivas integrales para abordar una serie de aspectos de muy diferente naturaleza, por ejemplo, la aplicación de conocimientos, el saber actuar bajo la guía de decisiones pertinentes o el despertar la conciencia y ser consecuentes con ella (Delors, 1996).

Las propuestas educativas actuales (MEC, 2015; CAA, 2007) reconocen como prioritario el desarrollo de la persona en sus diferentes dimensiones, y el aprendizaje durante toda la vida en el contexto de un mundo globalizado en cambio permanente (DeBoer, 2011).

Para Garritz (2009), numerosos aspectos, poco presentes en la educación más tradicional, son actualmente objetivos de primera fila en la enseñanza de las ciencias. Los cambios acelerados que caracterizan a los tiempos que vivimos demandan una actualización constante en la educación para adaptarla a los requerimientos de formar a una ciudadanía con capacidades para tomar decisiones y responder ante problemas relevantes que nos afectan a todos.

En el terreno de la formación científica y tecnológica, estos requerimientos demandan, entre otros aspectos, una enseñanza más humanizada y cercana a los intereses personales, éticos, culturales y políticos, cuyo estudio, además de estimulante y reflexivo, sirva para incrementar el pensamiento crítico. Se trata, en definitiva, de educar a través de la ciencia (UNESCO, 1993), lo cual, en el contexto de la sociedad actual, puede ser definido como Alfabetización Científica y Tecnológica (ACT, en adelante).

La ACT que facilite el ejercicio de una ciudadanía responsable requiere, según Hodson (2003), en cierto sentido, "politizar" el currículum de ciencia y tecnología, de modo que en él se incluya el análisis de algunos de los problemas que preocupan a la humanidad en la actualidad, promoviendo la contribución del alumnado en su propia formación para la acción responsable. Junto con la conveniencia de promover enfoques más interdisciplinares, Bybee y Fuchs (2006) destacan como elementos imprescindibles en esa ACT:

a) El pensamiento crítico;

b) Las habilidades de comunicación y de solución de problemas;

c) Los conocimientos, actitudes y valores que promuevan tomas de posturas y actuaciones responsables, tanto a nivel personal como social.

En la búsqueda de respuestas sobre como formar en la interdisciplinaridad y en las actitudes y los valores que esta sociedad cambiante demanda, cobra protagonismo el enfoque de enseñanza apoyado en problemas socio-científicos (Zeidler y otros, 2005) y, en ellos, la toma de decisiones 
informada, la capacidad de analizar, sintetizar y evaluar información, el razonamiento moral y los aspectos éticos (España y Prieto 2010). Autores como Kolstø (2001) y Edwards y otros (2004) señalan la pertinencia de este tipo de problemas para promover el pensamiento crítico, los valores democráticos y la participación activa en la búsqueda de vías de solución, a la par que el conocimiento científico necesario. En ellos, se ilustra la variedad y complejidad de los diferentes agentes implicados, lo cual favorece que los componentes personales y sociales del aprendizaje jueguen un papel relevante y potencialmente motivador (Sadler y Zeidler, 2005; Zeidler y otros, 2005).

Desde instancias oficiales (MEC, 2015; CAA, 2007), se suele proponer que el acercamiento a ellos realice a través de sus manifestaciones más cercanas y cotidianas al alumnado, como forma de despertar su interés por las materias científicas, y la conciencia social y humanitaria (Porlán, 2003). Éstos representan aspectos imprescindibles para que, en las actividades en el aula, adquieran protagonismo el razonamiento y la ética en la toma de decisiones (Develaki, 2008).

\section{La problemática energética}

El consumo y la producción de energía representa un claro ejemplo de problema sociocientífico (Martín, 2013). En su análisis y sus posibles vías de solución, inciden factores muy diversos asociados a:

- Factores medioambientales, ligados al impacto que el uso de determinadas fuentes de energía, como los combustibles fósiles o los componentes radiactivos, artífices de la energía nuclear, tienen en el medio natural.

- Factores políticos, ligados, entre otros, al elevado coste que la ciudadanía tiene que pagar por el disfrute de los mismos.

- Factores económicos, ligados a la preocupación por el mantenimiento de nuestro nivel de bienestar, el cual depende de unos recursos en peligro de agotamiento, frente a la gran disponibilidad de otros que, en el caso de unos adecuados avances tecnológicos, podrían explotarse a costes razonables.

Se trata de un asunto donde la controversia está presente, y que afecta tanto al dominio personal como al dominio social (Martín, Prieto y Jiménez, 2013). Aunque la temática sea una de las más enfatizadas en el currículum de ciencias, su tratamiento tradicional en poco parece contribuir a cambiar el pensamiento y la conducta de las personas en relación con el modelo energético predominante en nuestra sociedad (García y otros, 2007).

Un tratamiento alternativo necesario, que utilice como eje la comprensión de problemas reales en el mundo de hoy (Prieto, España y Martín, 2012), ha de implicar el aprendizaje conceptual, relacionado con la energía, e involucrar a las actitudes, los valores, los comportamientos y la conciencia de las personas.

\section{Las creencias en el profesorado de ciencias en la formación}

Los retos y desafíos que se presentan al profesorado de ciencias se encuentran en varios frentes. Respecto al contenido, es preciso admitir que los límites de sus disciplinas tradicionales, así como los enfoques para su enseñanza, se van ensanchando hacia espacios más interdisciplinares. Este ensanchamiento afecta igualmente al desarrollo de una gama más amplia de habilidades, actitudes y valores. Es decir, el profesorado tiene que asumir una concepción renovada de la educación, de su papel como docente, del papel del estudiante y de la manera de abordar la enseñanza-aprendizaje de las materias que constituyen su especialidad.

Lo que el profesorado cree y piensa, y como eso influye en lo que hace en el aula, representa una de las líneas de investigación en didáctica de las ciencias de más importancia en la actualidad (Porlán y 
otros, 1996; Porlán, y Martín del Pozo, 2004). Su influencia en las percepciones, los juicios y los comportamientos en el aula, son razones sobradas para identificarlas, reflexionar sobre ellas, comprenderlas y, tomar conciencia de las mismas y de sus efectos en los resultados de las innovaciones (Solís y Porlán, 2003; Porlán y Martín del Pozo, 2004).

Teniendo en cuenta estas consideraciones, nuestra implicación en la formación inicial de profesorado de ciencias nos ha llevado a reconocer la necesidad de indagar en la conciencia del futuro profesorado de ciencias sobre lo que representa la enseñanza de las ciencias en la actualidad, y su disposición a tener en cuenta los elementos antes considerados en las actividades formativas necesarias.

\section{Diseño de investigación}

El trabajo que aquí se presenta forma parte de un estudio más amplio, realizado en el contexto del programa formativo del Master de Profesorado de Ciencias de Educación Secundaria de la Universidad de Málaga, durante la docencia de la disciplina "Diseño y desarrollo de programaciones y actividades formativas" de las especialidades de "Física y Química" y "Biología y Geología”.

El estudio se ha centrado en la enseñanza y aprendizaje de la problemática energética, siendo uno de los ejes de incidencia el tratamiento del contenido científico en relación con la vida cotidiana, para que el alumnado pueda encontrarle el sentido con mayor facilidad (Solomon, 1992 y Aikenhead, 2006). Participaron un total de 29 profesores en formación inicial, 12 de ellos cursando la especialidad de "Física y Química", y 17 la de "Biología y Geología". El grupo estuvo compuesto por 17 mujeres y 12 hombres, con edades comprendidas entre los 22 y los 45 años.

En este artículo nos centramos en el significado que otorgan a la enseñanza y el aprendizaje de las ciencias en la actualidad y, en ella, a la inclusión de una serie de elementos cuya consideración es imprescindible en la formación inicial del profesorado de ciencias.

Hemos buscado dar respuesta a las siguientes preguntas: en las creencias de un grupo de profesorado de ciencias en formación inicial sobre la enseñanza y el aprendizaje de las ciencias, ¿̇en qué medida están presentes las nuevas tendencias que recomiendan el tratamiento de problemas socio-científicos en el aula de ciencias?; ¿qué importancia otorgan a los aspectos conceptuales en relación con aquellos que contribuyen al desarrollo de la conciencia?

Hemos buscado respuestas a las mismas a través de la aplicación de un cuestionario, para el diagnóstico de creencias, conformado por una parte cerrada, tipo Lickert, y otra abierta. Entre sus ítems, hemos seleccionado aquellos que inciden de manera más directa en los aspectos considerados en estas dos preguntas de investigación.

Según Pedretti y otros (2008), en un programa de formación inicial de profesorado de ciencias es preciso:

a) Estimular la reflexión sobre lo que significa la enseñanza y el aprendizaje de las ciencias, desafiar las nociones convencionales y considerar, de manera explícita, las ideologías de unos y otros enfoques de la enseñanza de las ciencias.

b) Delimitar nuevos enfoques y ponerlos en el lugar predominante que merecen ocupar en la enseñanza de las ciencias. Con este fin, consideran conveniente explorar sus posiciones sobre problemas controvertidos.

c) Actuar en sentido contrario al modelo de enseñanza más tradicional, transmisivo y apolítico, para promover, tras la toma de conciencia, la acción social. 
Nos basamos en la noción de un currículo de ciencias "socio-político" (Hodson, 2003; Roth y Barton 2004), que nos sugiere una visión de la enseñanza de las ciencias, donde la transformación y la acción, frente a la transmisión, se vuelven ejes para promover la responsabilidad ciudadana. El enfoque Ciencia-Tecnología-Sociedad (CTS), especialmente en su vertiente ambiental (CTSA) induce a valorar el potencial educativo de tratar problemas controvertidos en el aula, especialmente de la posibilidad de considerar simultáneamente valores alternativos (Pedretti y otros, 2008) y promover la participación cívica, la toma de decisiones y la acción responsable (Gonzalez y Prieto, 1998; Hodson, 2003; Zeidler y otros, 2005).

En torno a estas consideraciones y con aportaciones de la bibliografía, se fueron configurando proposiciones del cuestionario que conformaron las afirmaciones tipo Likert (Creswell y Plano-Clark, 2007). Ante cada proposición, los participantes debían de mostrar su grado de acuerdo en un rango de respuesta comprendido entre 1-4. En esta decisión se excluyó la opción neutral para evitar opciones que pudieran ser interpretables como falta de conocimiento, indiferencia o falta de motivación. La formulación de las afirmaciones y de su contenido fue sometida a la valoración y análisis de un grupo de 7 expertos en el área de Didáctica de las Ciencias Experimentales. Una prueba piloto en una muestra de 49 estudiantes de la Licenciatura de Pedagogía de $4^{\circ}$ curso, nos sirvió para comprobar que eran comprendidos adecuadamente. Los 5 ítems considerados en este trabajo están recogidos en el cuadro 1.

Tabla 1

Ítems tipo Lickert

\begin{tabular}{|c|l|}
\hline ÍTEMS & \multicolumn{1}{c|}{ ENUNCIADOS } \\
\hline Ítem $\mathbf{1}$ & $\begin{array}{l}\text { En la actualidad resulta prácticamente imposible discutir sobre valores humanos y } \\
\text { problemas políticos y económicos sin recurrir al papel que juegan en ellos la ciencia y } \\
\text { la tecnología. }\end{array}$ \\
\hline Ítem 2 & $\begin{array}{l}\text { Para promover la alfabetización científica y tecnológica no es preciso tener en cuenta } \\
\text { consideraciones morales y éticas, actitudes y valores. }\end{array}$ \\
\hline Ítem 3 3 & $\begin{array}{l}\text { En el aula de ciencias, es contraproducente que se trabajen problemas reales (como, } \\
\text { por ejemplo, el de la energía), porque demandan consideraciones políticas, éticas y } \\
\text { económicas, que distraerían a los alumnos de lo que tienen que aprender. }\end{array}$ \\
\hline Ítem $\mathbf{4}$ & $\begin{array}{l}\text { En la enseñanza de las ciencias es preciso poner énfasis en problemas reales y } \\
\text { socialmente relevantes que tienen que ver con la ciencia y la tecnología. }\end{array}$ \\
\hline Ítem 5 & $\begin{array}{l}\text { Debido a que los problemas que enfrenta nuestro tiempo no son exclusivos de la } \\
\text { ciencia, es necesario el dialogo y el intercambio de información multidisciplinar en la } \\
\text { enseñanza de las ciencias. }\end{array}$ \\
\hline
\end{tabular}

La propuesta $\mathrm{n}^{\circ} 1$ se dirige a poner de manifiesto, a través de su nivel de acuerdo con ella, el grado de reconocimiento de la influencia que factores políticos, económicos y sociales tienen sobre la ciencia y la tecnología (Bouillon y Gómez, 2001 y Brown, Lauder y Ashton, 2008), y la necesidad de tenerlos en cuenta en la enseñanza.

Mediante la propuesta $n^{\circ} 2$ nos aproximamos al grado de aceptación del hecho de que conseguir una formación científica básica adecuada en el alumnado conlleva el desarrollo de ciertas actitudes y, valores éticos y morales, los cuales deben promoverse desde el aula. La propuesta $\mathrm{n}^{\circ} 3$ busca recabar de los participantes su grado de acuerdo con las tendencias a trabajar las vertientes más sociales del problema (Holbrook y Rannikmäe, 2009). 
Con la propuesta $\mathrm{n}^{\circ} 4$ tratamos de que los participantes muestren su posicionamiento respecto al enfoque de centrar la enseñanza en problemas socio-científicos (Hurd, 1998; Pedretti y otros, 2008; Develaki, 2008; España y Prieto, 2010; DeBoer, 2011).

Con la propuesta $n^{\circ} 5$, esperamos que los participantes nos muestren el grado en que reconocen que la enseñanza de las ciencias requiere la consideración de conocimientos procedentes de otras disciplinas (Lemke, 2006; Garritz, 2009).

Las preguntas abiertas han sido formuladas con el objetivo de ampliar la recogida de información, dando la oportunidad a los participantes de centrarse en los aspectos que más les importan o preocupan, y de conocer la relevancia que otorgan a la problemática energética como contexto de enseñanza. Para contextualizarlas, se propuso la lectura previa de fragmentos elegidos del contenido de un capítulo de un libro de texto dedicado a la energía en $2^{\circ}$ curso de la ESO de la asignatura "Ciencias de la Naturaleza". El nivel de $2^{\circ}$ de la ESO fue elegido por tratarse de contenidos comunes para todo el alumnado receptor del objetivo de formación científica para ejercer una ciudadanía participativa y responsable.

En él se abordan, a modo de contenidos, la definición de fuente de energía, de energía primaria y energía secundaria, de fuentes de energía renovables y no renovables y su disponibilidad futura. Asimismo, aparecen algunas ventajas e inconvenientes de las diferentes fuentes de energía y de sus usos, las ventajas del ahorro y la eficiencia energética. Las preguntas fueron dos:

1. ¿Crees que es importante abordar el problema de la energía en el aula de ciencias? Justifica tu respuesta.

2. En el texto se desarrollan dos aspectos: a) Conceptos relacionados con la energía y b) el problema de la energía, ¿Cuál crees debería tratarse en mayor medida? Justifica tu respuesta.

\section{Análisis de datos y resultados}

El análisis de las respuestas a las preguntas tipo Likert se inició otorgando a cada una de las opciones una tendencia: "muy innovadora" (T1), "innovadora" (T2), "tradicional" (T3) y "muy tradicional" (T4).

Tabla 2

Frecuencias en las tendencias ante cada uno de los items

\begin{tabular}{|c|c|c|c|c|c|}
\hline Tendencia & Ítem 1 & Ítem $\mathbf{2}$ & Ítem 3 & Ítem $\mathbf{4}$ & Ítem 5 \\
\hline T1 & 4 & 17 & 22 & 16 & 11 \\
\hline T2 & 13 & 10 & 5 & 12 & 18 \\
\hline T3 & 9 & 1 & 2 & 1 & 0 \\
\hline T4 & 3 & 1 & 0 & 0 & 0 \\
\hline
\end{tabular}

En la tabla 1 se recogen las frecuencias de estas tendencias en cada uno de los ítems. En ella se aprecia la predominancia de las tendencias muy innovadora (T1) e innovadora (T2) en los ítems 25. Es en el ítem 1 donde se concentra un mayor número de participantes en las tendencias tradicional $(\mathrm{T} 3, \mathrm{~F}=9)$ y muy tradicional $(\mathrm{T} 4, \mathrm{~F}=3)$. Sobre este dato, y a través de entrevistas informales al profesorado en formación (pf), pudimos apreciar que reconocen el importante papel que juegan la ciencia y la tecnología en todas las parcelas de la sociedad, pero algunos se mostraron reticentes con el hecho de que para discutir sobre valores humanos y, problemas políticos y 
económicos, se tenga que recurrir, de manera inevitable, al papel que juegan en ellos la ciencia y la tecnología.

En algunos, además, se aprecia cierto desacuerdo con lo que en el ítem se afirma: “(...) yo creo que te pueden preocupar los problemas políticos sin tener que asociarlos a la ciencia y la tecnología" (pf.19)

En este punto, detectamos una necesidad de centrar la atención formativa, en el sentido de poner de manifiesto y promover la conciencia de la ciencia y la tecnología como protagonistas claves en el debate sobre numerosas cuestiones sociales.

Las respuestas ante los ítems 2 y 3 ponen de manifiesto que la introducción de las implicaciones morales y éticas, las actitudes y los valores en el tratamiento de problemas reales son elementos muy bien considerados, en general. Esto nos sugiere que, de partida, no sería preciso prestar una atención específica a la promoción de este aspecto, si bien, de nuevo, las conversaciones informales nos indicaron que conviene hacer un seguimiento de la convicción que muestran sobre el mismo: "pero no olvidemos que, para trabajar los valores, tenemos que asegurarnos que el alumnado ha comprendido los conceptos básicos" (pf.16)

Respecto a la importancia de los problemas socio-científicos en la enseñanza de las ciencias (ítem 3) y la apertura a la interdisciplinaridad (item 5), los resultados muestran un alto grado de acuerdo, así como con la educación en actitudes y valores, las consideraciones de tipo político, ético y económico, y la controversia que todos estos elementos conllevan.

El segundo paso en el análisis de las respuestas ha consistido en combinar las tendencias mostradas en cada uno de los cinco ítems para definir cuatro perfiles, que hemos considerado indicadores de sus posturas hacia la innovación que se propone. El significado que hemos otorgado a dichos perfiles es el siguiente:

- Perfil 1 (P1): "Muy buena tendencia a la innovación”. En él se agrupan los casos en los que, al menos ante 4 ítems, se muestra la postura muy innovadora (T1) y ante el quinto la postura innovadora (T2).

- Perfil 2 (P2): "Buena tendencia a la innovación". A él se han asignado los casos en los que las respuestas aparecen repartidas entre las tendencias 1 y 2 (no más de tres en la T1).

- Perfil 3 (P3): “Aceptable tendencia a la innovación”. Este perfil se ha asignado a los casos en los que se presentan tres opciones en las tendencias 1 o 2 y las otras dos en las tendencias 3 o 4.

- Perfil 4 (P4): “Baja tendencia a la innovación”. Este perfil le corresponde a aquellos casos en los que aparecen tres opciones, como mínimo, en las tendencias 3 o 4.

Procediendo de esta manera, a cada participante le corresponde un solo perfil, que puede ser: P1, P2, P3 o P4. 


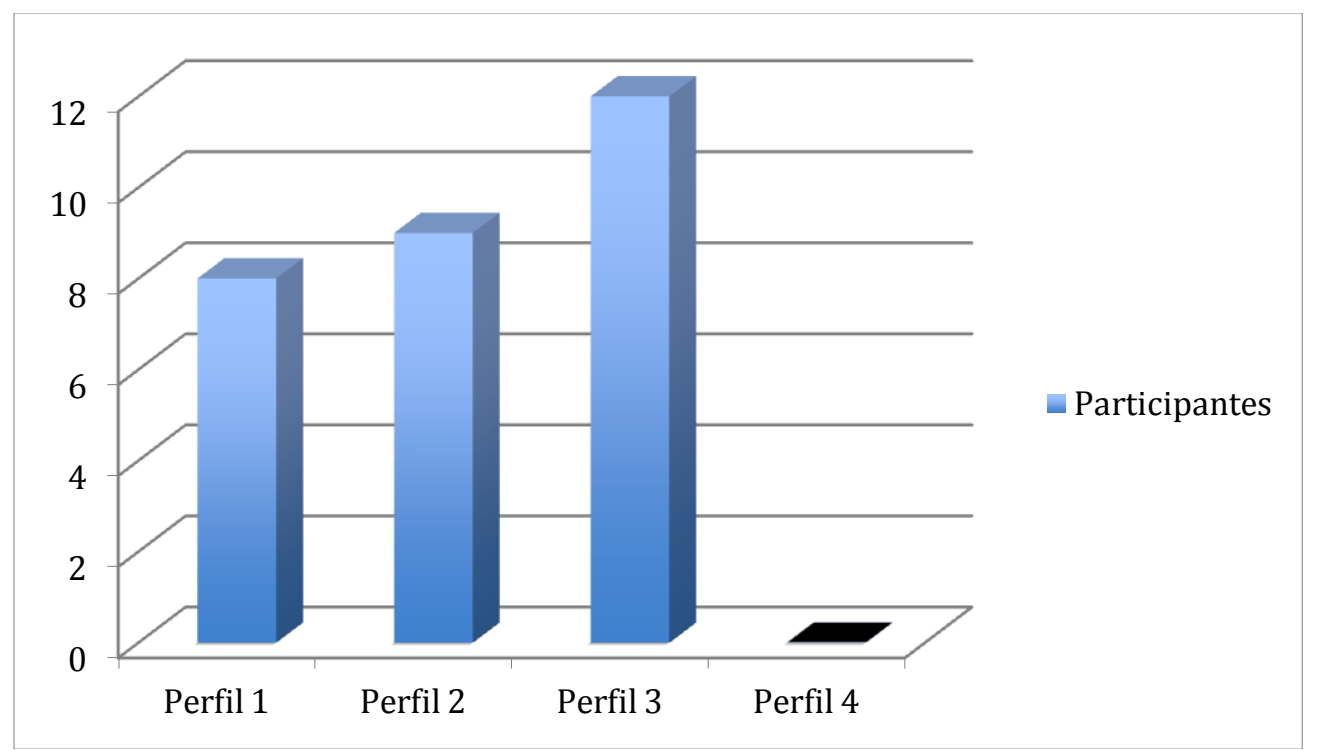

Gráfico 1. Perfiles de los participantes

En el Gráfico 1 se recoge la presencia de los diferentes perfiles. En él puede observarse el alto grado de aceptación de las propuestas que les hemos formulado, ya que la mayoría (17) se ubica en los perfiles que se corresponden con la tendencia "Muy buena" (8 en P1) y con la tendencia "Buena" (9 en P2) hacia la innovación. Las posturas de 12 de los participantes reflejan un perfil 3, relativo a una "Aceptable" tendencia a la innovación, y es positivo el hecho de que ninguno se ubique en el perfil de baja tendencia a la innovación.

Es decir, nuestro profesorado en formación inicial se ven a sí mismos como partidarios de la innovación que les proponemos, con más o menos matices.

A continuación, en el análisis de las preguntas abiertas, que nos ofrece el discurso propio de los participantes, se ha aplicado un proceso de categorización abierta de las ideas contenidas (Bliss, Monk y Ogborn, 1983). Las tres categorías no excluyentes formuladas a partir del contenido de las respuestas a la pregunta 1 son las siguientes:

- Categoría 1A. Contenidos conceptuales.

- Frecuencia: $\mathrm{F}=16$

- Contenido: aprendizaje de los conceptos relacionados con el tema, antes de abordar la problemática energética.

○ Ejemplo: "En primer lugar, es importante que los alumnos tengan conocimiento de los tipos de energía existentes y de su uso...” (pf. 16)

- Categoría 1B. Actitudes, valores y conciencia.

- Frecuencia: $\mathrm{F}=20$

- Contenido: actitudes, valores y conciencia ante los problemas sociales y actuaciones individuales para contribuir a su solución (responsabilidad ciudadana).

○ Ejemplo: “...que aprendan a ahorrar energía y cuidar el medioambiente.” (pf. 28)

- Categoría 1C. Pensamiento crítico y toma de decisiones.

- Frecuencia: $\mathrm{F}=11$

- Contenido: desarrollo del pensamiento crítico, y su aplicación en la obtención de conclusiones y en la toma de decisiones.

○ Ejemplo: “(...)fomentar una actitud crítica ante el gasto desmesurado...” (pf.15). 
El desarrollo en el alumnado de actitudes, valores y conciencia ciudadana representa la razón con más presencia (20 participantes), a la hora de decidir abordar la problemática energética en el aula.

Estas consideraciones van, con frecuencia, acompañadas de otras en las que se destaca la importancia de trabajar la problemática energética como vía para aprender conceptos científicos. Los 16 participantes que lo hacen, consideran, al mismo tiempo, el potencial del problema en el desarrollo de actitudes, valores y toma de conciencia: "por supuesto que sí; abordar el problema de la energía supone, por un lado, acercar a los alumnos los conceptos con los que están trabajando así como mejorar su interactividad en clase, y por otro lado, conocer los problemas globales les hace tomar conciencia ante la vida, les proporciona herramientas para conocer donde viven y les hace ciudadanos responsables." (pf. 18), (cat. 1A y 1B).

Por otro lado, la promoción del espíritu crítico en el pensamiento y en la toma de decisiones, es considerada por 11 de los participantes, que lo hacen junto con otro tipo de consideraciones. Ejemplo de ello es la siguiente respuesta dada: "sí, las fuentes energéticas mayoritarias actualmente suponen bastantes inconvenientes (reservas limitadas, peligrosidad, contaminación...) por lo que es necesario un cambio en la gestión de la energía, que debe ir unido a un cambio en la mentalidad de la población, incluyendo a niños y adolescentes que deben ser conscientes de la situación actual para poder sacar sus propias conclusiones." (pf. 2) (cat. 1B y 1C).

En el análisis de las respuestas a la segunda pregunta abierta, se ha cuantificado el número de participantes en cada una de las opciones: a), b) o ambas, en la primera parte, y categorizado los argumentos justificativos, en la segunda.

En términos generales, una amplia mayoría (22) dedicaría más tiempo al tratamiento del problema que a los conceptos relacionados; 5 participantes darían igual tratamiento a ambos aspectos y 2 priorizarían los conceptos básicos relacionados.

En cuanto a categorías en las razones alegadas y sus frecuencias, se aprecian las siguientes:

- Categoría $2^{\text {a }}$. Explicar primero los conceptos científicos.

- Frecuencia: $\mathrm{F}=15$

- Contenido: imposibilidad de entender la problemática energética sin haber comprendido los conceptos relacionados.

- Ejemplo: "Primero deben de entender que es la energía, origen de las energías, tipos de energía, usos, y finalmente los problemas que se crean" (pf. 3)

- Categoría 2B. Introducir los problemas para entender los conceptos.

- Frecuencia: $\mathrm{F}=2$

- Contenido: la comprensión de los conceptos relacionados con la problemática puede verse favorecida mediante el tratamiento contextualizado en la misma.

- Ejemplo: "Educar al alumno en el uso responsable de los recursos energéticos, además de hacerles conscientes de cómo interacciona ciencia y tecnología con sociedad, economía y política. A medida que van entendiendo esto irán asimilando los conceptos relacionados." (pf. 5).

- Categoría 2C. Invitar a la reflexión y la crítica

- Frecuencia: $\mathrm{F}=$

- Contenido: El principal criterio para priorizar uno u otro aspecto es el fomento de la reflexión y el pensamiento crítico.

- Ejemplo: 'Debería tratarse en mayor medida el problema de la energía aplicando un razonamiento en un marco social y promoviendo que desarrollen un pensamiento crítico y razonado." (pf. 14)

- Categoría 2D. Concienciar sobre el problema y sus soluciones.

- Frecuencia: $\mathrm{F}=18$ 
- Contenido: Prima la necesidad de promover la conciencia y despertar el interés hacia la problemática y las posibles actuaciones para mejorarla.

○ Ejemplo:"El alumno debe saber que él también es parte del problema porque son el futuro y ellos van a heredar el problema." (pf. 6)

De la amplia mayoría (22) que le dedicaría más tiempo al tratamiento del problema que a los conceptos relacionados, 9 considera que sería necesario trabajar antes los conceptos "básicos" para poder entender el problema (cat. 2A). También se manifiestan en el mismo sentido 4 de los 5 participantes que darían igual tratamiento a ambos aspectos. Es decir, si bien 27 consideran importante dedicar un tiempo mayor o similar a tratar la problemática energética que a los conceptos relacionados, 13 especifican que no se puede llegar a trabajar el problema si antes no se han comprendido dichos conceptos: “ambos por igual. Son dos aspectos que están ligados. No puede comprenderse el problema de la energía sin conocer primero los conceptos relacionados, pero tampoco es conveniente un aprendizaje mecánico de los conceptos sin estimular el sentido crítico y el interés por la actualidad de nuestro mundo" (pf. 7) (cat. 2A, 2C y 2D).

Los 14 participantes restantes, que tratarían en mayor medida la problemática energética, enfatizan el propósito de promover la toma de conciencia ante el problema y sus posibles soluciones, sobre la base de que estas ejercen una importante motivación en el alumnado a la hora de aprender los conceptos (cat. 2D): "el problema de la energía. Aunque tienen que llegar a comprender los conceptos y generalidades de la energía, el punto más importante es el que repercute directamente sobre la población. Somos causantes, partícipes y receptores de las causas y efectos derivados del uso y también del despilfarro de la energía, así como de los efectos.” (pf. 15) (cat. $2 \mathrm{~A}$ y $2 \mathrm{D}$ )

Para dos de los participantes, el tratamiento del problema representa, ya en sí, un mecanismo para el aprendizaje de los conceptos implicados (cat. 2B): "debemos educar al alumno en uso responsable de los recursos energéticos, además de hacerles conscientes de cómo se interacciona ciencia y tecnología con sociedad, economía y política. A medida que van entendiendo esto, irán asimilando los conceptos relacionados con la energía." (pf. 5) (cat. 2B y 2D)

\section{Implicaciones y consideraciones finales}

Lo primero que nos llama la atención es la alta disposición de los participantes a la innovación que les proponemos. No obstante, esta se va matizando a medida que van exponiendo y ampliando sus ideas.

\section{Interdisciplinaridad}

Aquí, las matizaciones guardan relación con una concepción y una aproximación al tratamiento interdisciplinar en el que predomina la tendencia a considerar a las ciencias como materias aisladas de las cuestiones sociales, y donde no se suele asociar el debate político y social a la ciencia y la tecnología.

En este caso, es necesario insistir, mejor a base de ejemplos, en las interrelaciones cienciapolítica, ciencia-economía, entre otras. Sólo así, evitaremos que el tratamiento interdisciplinar al que se alude se quede centrado en la consideración de aspectos parciales y concretos derivados de los impactos en el medio. También resulta muy aconsejable poner de manifiesto las consecuencias potenciales de obviar la presencia de la ciencia y la tecnología en el tratamiento de determinados problemas económicos y políticos de actualidad. 


\section{Conciencia}

La toma de conciencia aparece reconocida en alto grado, al igual que las actitudes y los valores. La pregunta: ¿conciencia, actitudes y valores sobre qué?, aparece con respuesta difusa en cuanto a su alcance, su ubicación y la manera de promoverla. Puede relacionarse con el hecho de que no se considere imprescindible introducir a la ciencia y la tecnología en los grandes debates económicos y políticos de nuestro tiempo.

No es preciso, por tanto, estimular el tratamiento de actitudes, valores, etc., ya que el profesorado en formación inicial les otorga un gran protagonismo, sino abrir formas y caminos para su tratamiento adecuado e integrado. De esta manera, también se estará contribuyendo a integrar a las ciencias con el resto de las disciplinas.

\section{Aprendizaje conceptual}

Las llamadas al aprendizaje conceptual aparecen como requisito previo, y mucho menos como algo que puede ocurrir en el camino de la toma de conciencia sobre una determinada problemática. Tiene una gran presencia el modelo, según el cual, los conceptos se pueden aprender en abstracto, sin aludir a su utilidad para explicar determinados problemas, los cuales no se podrán entender si los conceptos no han sido abordados. De esa manera, se da por sentado, de forma implícita, que el enfoque innovador no conlleva el aprendizaje de los conceptos en sí. Aquí aparece una paradoja: a) los debates pueden tener lugar sin la Ciencia y la Tecnología, y b) para llevar el debate socio-científico al aula es preciso enseñar primero los conceptos. En ambos casos, y aunque esto ocurre a la par que se muestran altos grados de aceptación de propuestas innovadoras, el pensamiento está actuando en contra de la presencia de los problemas socio-científicos en el aula. En estos casos, hay que prestar atención, no tanto a lo positivo en que son recibidas las propuestas innovadoras, sino a la variedad de condicionantes que se suceden tras la aceptación inicial, ya que son ellos los que nos ofrecen pistas para comprender por qué determinadas innovaciones no fueron llevadas a la práctica al final. Así, matizaciones realizadas tras un alto grado de apoyo a la introducción de problemas socio-científicos, a la promoción de valores y actitudes y al tratamiento de los aspectos interdisciplinares, suelen venir referidas al enfoque de enseñanza que se adopte. Y a veces, el aprendizaje de conceptos se suele enfrentar al desarrollo de la innovación.

Estos resultados nos indican la necesidad de poner énfasis, en la formación inicial de profesorado de ciencias, en intervenciones basadas en casos de problemas socio-científicos. En ellos, se ha de poner en evidencia la posibilidad de un acercamiento interdisciplinar a la enseñanza de las ciencias y la tecnología, que, a su vez facilite la integración de experiencias cercanas y cotidianas a las que el alumnado otorgue importancia.

Tal enfoque también ha de fomentar la reflexión, el pensamiento crítico, la responsabilidad y la motivación hacia la acción en el alumnado, ya que la valoración de estos aspectos como pilares fundamentales en el progreso hacia la alfabetización científica y tecnológica del alumnado no han aparecido priorizados.

\section{Referencias}

Aikenhead, G.S. (2006). Science education for everyday life: Evidence-based practice. New York: Teachers College Press.

Bliss, J., Monk, M. \& Ogborn, J. (1983). Qualitative data analysis for educational research. London: CroomHelm. 
Bouillon, L.M. \& Gómez, L.M. (2001). Connecting school and community with science learning: Real world problems and school-community partnerships as contextual scaffolds. Journal of Research in Science Teaching, 38(8), 878-898.

Brown, P., Lauder, H. \& Ashton, D. (2008). Education, Globalisation and the Future of the Knowledge Economy. European Educational Research Journal, 7(2), 131-56.

Bybee, R.W. \& Fuchs, B. (2006). Preparing the 21st Century Workforce: A New Reform in Science and Technology Education. Journal of Research in Science Teaching, 43(4), 349-352.

CAA (2007). Orden de agosto de 2007, por la que se desarrolla el currículo correspondiente a la Educación Secundaria Obligatoria en Andalucía. (BOJA 5 de enero de 2007).

Creswell, J.W. \& Plano-Clark, V.L. (2007). Designing and conducting mixed methods research. Thousand Oaks, CA: Sage Publications.

Deboer, G.E. (2011). The Globalization of Science Education. Journal of Research in Science Teaching, $48(6), 567-591$.

Delors, J. (1996). La educación encierra un tesoro. México: Ediciones UNESCO.

Develaki, M. (2008). Social and ethical dimension of the natural sciences, complex problems of the age, interdisciplinarity, and the contribution of education. Science \& Education, 17, 873-888.

Edwards, M., Gil, D., Vilches, A. \& Praia, J. (2004). La atención a la situación del mundo en la educación científica. Enseñanza de las Ciencias, 22(1), 47-64.

España, E. \& Prieto, T. (2010). Los problemas socio-científicos como contexto para la enseñanza y el aprendizaje de las ciencias. Investigación en la Escuela, 71, 17-24.

García, J.E., Rodríguez, F., Solís, M.C. \& Ballenilla, F. (2007). Investigando el problema del uso de la energía. Investigación en la Escuela, 63, 29-45.

Garritz, A. (2009). La enseñanza de la ciencia en una sociedad con incertidumbre y cambios acelerados. Conferencia Inaugural del VIII Congreso Internacional sobre Investigación en la Didáctica de las Ciencias, Barcelona, 7 septiembre 2009.

González, F.J. \& Prieto, T. (1998). Educar para la democracia. La Ciencia-Tecnología-Sociedad. Investigación en la escuela, 34, 59-67.

Hodson, D. (2003). Time for action: Science education for an alternative future. International Journal of Science Education, 25(6), 645-670.

Holbrook, J. \& Rannikmäe, M. (2009). The meaning of scientific literacy. International Journal of environmental \& Science Education, 4(3), 275-288.

Hurd, P.D. (1998). Scientific literacy: New minds for a changing world. Science Education, 82, 407-416.

Kolstø, S.D. (2001). Scientific literacy for citizenship: Tools for dealing with the science dimension of controversial socio-scientific issues. Science Education, 85, 291-310.

Lemke, J.L. (2006). Investigar para el futuro de la educación científica: Nuevas formas de aprender, Nuevas formas de vivir. Enseñanz̧a de las Ciencias, 24(1), 5-12.

Martín, C. (2013). La problemática energética como contexto de enseñanza-aprendizaje: una experiencia con profesorado de Ciencias en formación inicial. Tesis inédita de Doctorado. Universidad de Málaga Málaga: Riuma. Recuperado de http://hdl.handle.net/10630/5597

Martín, C., Prieto, T. \& Jiménez, M.A. (2013). El problema de la producción y el consumo de energía: ¿Cómo es tratado en los libros de texto de Educación Secundaria? Enseñanza de las ciencias, 30(3), 153-171.

MEC. (2015). Real Decreto 1105/2014, de 26 de diciembre, por el que se establece el currículo básico de la Educación Secundaria Obligatoria y del Bachillerato (BOE 3 de enero de 2015).

Pedretti, E.G., Bencze, L., Hewitt, J., Romkey L. \& Jivraj, A. (2008). Promoting Issues-based STSE Perspectives in Science Teacher Education: Problems of Identity and Ideology. Science \& Education, 17, 941-960. 
Porlán, R. (2003). Principios para la Formación de Profesores de Secundaria. Revista Interuniversitaria de Formación del Profesorado, 17(1), 23-35.

Porlán, R., Azcárate, P., Martín Del Pozo, R., Martín, J. \& Rivero. A. (1996). Conocimiento profesional deseable y profesores innovadores: fundamentos y principios formativos. Investigación en la Escuela, 29, 23-38.

Porlán, R. \& Martín Del Pozo, R. (2004). The conceptions of inservice and prospective primary school teachers about the teaching and learning of science. Journal of Science Teacher Education, 15, 39-62.

Prieto, T., España, E. \& Martín, C. (2012). Algunas cuestiones relevantes en la enseñanza de las ciencias desde una perspectiva Ciencia-Tecnología-Sociedad. Revista Eureka sobre Enseñanza y Divulgación de las Ciencias, 9(1), 71-77.

Roth, W.M. \& Barton, A.C. (2004). Rethinking scientific literacy. New York: Routledge Falmer.

Sadler, T.D. \& Zeidler, D.L. (2005). Patterns of informal reasoning in the context of socioscientific decision making. Journal of Research in Science Teaching, 42(1), 112-138.

Solís, E. y Porlán, R. (2003). Las concepciones del profesorado de ciencias de secundaria en formación inicial: obstáculos o punto de partida. Investigación en la Escuela, 49, 5-22.

Solomon, J. (1992). The classroom discussion of science-based social issues presented on television: Knowledge, attitudes and values. International Journal of Science Education, 14, 431-444.

UNESCO. (1993). International forum on scientific and technological literacy for all. Final report. Recuperado de http://unesdoc.unesco.org/images/0009/000952/095246eb.pdf

Zeidler, D.L., Sadler, T.D., Simmons, M.L. \& Howes, E.V. (2005). A research based framework for socio-scientific issues education. Science Education, 89(3), 357-377.

\section{Sobre los Autores}

Nombre: Carolina Martín Gámez

Institución: Universidad de Sevilla

E-mail: cmartin17@us.es

Información biográfica: Profesora Ayudante Doctora en el Departamento de Didáctica de las Ciencias Experimentales y Sociales. Facultad de Ciencias de la Educación.

ORCID: no disponible

Nombre: Teresa Prieto Ruz

Institución: Universidad de Málaga

E-mail: ruz@uma.es

Información biográfica: Catedrática de Universidad en el Departamento de Didáctica de la Matemática, las Ciencias Sociales y las Ciencias Experimentales. Facultad de Ciencias de la Educación.

ORCID: no disponible

Nombre: Ma Ángeles Jiménez López

Institución: Universidad de Málaga

E-mail: majimenez@uma.es

Información biográfica: Titular de Universidad en el Departamento de Didáctica de la Matemática, las Ciencias Sociales y las Ciencias Experimentales. Facultad de Ciencias de la Educación.

ORCID: no disponible 


\section{Investigación en la Escuela}

Revista académica evaluada por pares y de acceso abierto

Número 88

1 de octubre de 2016

ISSN 2443-9991

\section{(c)}

SOMERIIGHISRESERVED Los/as lectores/as pueden copiar, mostrar, y distribuir este artículo, siempre y cuando se de crédito y atribución al autor/es y a Investigación en la Escuela, se distribuya con propósitos no-comerciales, no se altere o transforme el trabajo original. Más detalles de la licencia de Creative Commons se encuentran en http://creativecommons.org/licenses/by-nc-sa/3.0 Cualquier otro uso debe ser aprobado en conjunto por el autor/es, o Investigación en la Escuela.

Contribuya con comentarios y sugerencias en la web de la revista. Por errores y sugerencias contacte a investigacionescuela@ddcc.uhu.es 


\section{Investigación en la escuela}

Consejo de dirección: Ana Rivero García (Universidad de Sevilla), Nicolás de Alba Fernández (Universidad de Sevilla), Pedro Cañal de León (Universidad de Sevilla), Francisco F. García Pérez (Universidad de Sevilla), Gabriel Travé González, (Universidad de Huelva), Francisco F. Pozuelos Estrada (Universidad de Huelva)

\section{Dirección: Ana Rivero García y Nicolás de Alba Fernández}

Técnico de edición: Francisco Javier López Sánchez

\section{Consejo editorial}

José Félix Angulo Rasco. Universidad de Cádiz Rosa Ma Ávila Ruiz. Universidad de Sevilla Pilar Azcárate Goded. Universidad de Cádiz Juan Bautista Martínez Rodríguez. Universidad de Granada

Nieves Blanco García. Universidad de Málaga Fernando Barragán Medero. Universidad de La Laguna José Carrillo Yáñez. Universidad de Huelva José Contreras Domingo. Universidad de Barcelona. Luis C. Contreras González. Universidad de Huelva Ana $\mathbf{M}^{\mathbf{a}}$ Criado García-Legaz. Universidad de Sevilla Rosario Cubero Pérez. Universidad de Sevilla José $\mathbf{M}^{\mathbf{a}}$ Cuenca López. Universidad de Huelva Jesús Estepa Giménez. Universidad de Huelva Rafael Feito Alonso. Universidad Complutense (Madrid)

Francisco José García Gallardo. Universidad de Huelva

Soledad García Gómez. Universidad de Sevilla J. Eduardo García Díaz. Universidad de Sevilla
Fernando Hernández Hernández. Universidad de Barcelona

Salvador Llinares Ciscar. Universidad de Alicante Alfonso Luque Lozano. Universidad de Sevilla Rosa Martín del Pozo. Universidad Complutense (Madrid)

José Martín Toscano. IES Fernando Herrera (Sevilla) Jaume Martínez Bonafé. Universidad de Valencia F. Javier Merchán Iglesias. Universidad de Sevilla Emilia Moreno Sánchez. Universidad de Huelva. Rosario Ortega Ruiz. Universidad de Córdoba Antonio de Pro Bueno. Universidad de Murcia Fco. de Paula Rodríguez Miranda. Universidad de Huelva

Pedro Sáenz-López Buñuel. Universidad de Huelva Antoni Santisteban Fernández. Universidad Autónoma (Barcelona)

Emilio Solís Ramírez. Catedrático de IES. $\mathbf{M}^{\mathbf{a}}$ Victoria Sánchez García. Universidad de Sevilla. Magdalena Suárez Ortega. Universidad de Sevilla

\section{Consejo asesor}

Manuel Area Moreira. Universidad de La Laguna

Jaume Carbonell. Director Cuadernos de Pedagogía. Barcelona

César Coll. Universidad de Barcelona

Christopher Day. Universidad de Nothingham. U.K.

Juan Delval. Universidad Nacional de Educación a Distancia

John Elliott. Universidad de East Anglia. Norwich. U.K.

José Gimeno Sacritán. Universidad de Valencia

André Giordan. Universidad de Paris VII y Ginebra

Francisco Imbernón. Universidad de Barcelona

Ángel Pérez Gómez. Universidad de Málaga

Rafael Porlán Ariza. Universidad de Sevilla

Francesco Tonucci. Instituto de Pedagogía del C.N.R. Roma

Jurjo Torres Santomé. Universidad de A Coruña 